\title{
Re-print Non-aspirin NSAIDs use is associated with lowering of liver fibrosis scores in patients with fatty liver disease
}

\author{
Ikechukwu Ogbu ${ }^{*}$, Hossein Akhondi MD ${ }^{2}$, Napatkamon Ayutyanont $\mathrm{PhD}^{3}$, Andre Manov $\mathrm{MD}^{4}$ \\ ${ }^{1}$ Internal Medicine Department, Mountain View Hospital Sunrise Health Consortium, Las Vegas, NV. \\ ${ }^{2}$ Associate Program Director, Mountain View Hospital Associate Professor of Medicine, University of Nevada. \\ ${ }^{3}$ Division Director of Research,HCA Far West Division, Las Vegas, NV. \\ ${ }^{4}$ Associate Program Director, Mountain View Hospital, Sunrise Health Consortium, Las Vegas, NV, Professor of Medicine, TCU and UNT School of \\ medicine, Fort Worth, TX.
} *Corresponding Author: Ikechukwu Ogbu, Internal Medicine Department, Mountain View Hospital Sunrise Health Consortium, Las Vegas,
NV.

Received Date: 15 April 2021 | Accepted Date: 26 July 2021 | Published Date: 30 July 2021

Citation: I Ogbu, H Akhondi, N Ayutyanont, A Manov. (2021) Non-aspirin NSAIDs use is associated with lowering of liver fibrosis scores in patients with fatty liver disease. Endocrinology and Disorders. 5(6): DOI:10.31579/2640-1045/071

Copyright: (C) 2021 Ikechukwu Ogbu. This is an open-access article distributed under the terms of the Creative Commons Attribution License, which permits unrestricted use, distribution, and reproduction in any medium, provided the original author and source are credited.

\begin{abstract}
:
Only a few studies are available with appropriate data on the effects of non-aspirin, non-steroidal anti-inflammatory drugs (NSAID's) use in patients with fatty liver disease. We performed a retrospective study of 1347 patients with imaging studies that showed fatty liver disease from 2016 through 2019.

Then we determined the change in validated indices using Fibrosis-4 (FIB4) and NAFLD fibrosis score (NFS). Patient's clinical information, including NSAID's use, was collected at baseline and then yearly. Using generalized linear model, we estimated the association between the non-aspirin NSAID's use and change in the baseline indices. Non-aspirin NSAID's use was found to be associated with significant lowering of FIB-4 score $(0.596$ units lower, p-value $<0.0001)$ and NFS score (0.431 units lower, p-value 0.0027$)$ every year.

In this retrospective study of patients with fatty liver disease found on imaging, non-aspirin NSAID's use was associated with lowering of fibrotic scores, suggesting that non- aspirin containing NSAID's use might be associated with a lower risk for advanced fibrosis in patients with fatty liver disease.

Summary: Non-aspirin NSAID's use is associated with lowering of hepatic fibrosis scores in patients with fatty liver disease.

Key Words: fatty liver disease; NSAID's; aspirin, ibuprofen; liver fibrosis; advance liver disease; cirrhosis; liver fibrosis indices; NAFLD score; Fibrosis-4 score
\end{abstract}

\section{Introduction}

Fatty liver disease is defined as the presence of fatty infiltration of the liver. Over time, about $10-25 \%$ of patients with fatty liver disease will progress to advanced fibrosis, cirrhosis, hepatocellular carcinoma, and death [1]. The gold standard to diagnose fatty liver disease and fibrosis is by liver biopsy. However, because this method is invasive, some validated scoring systems are often used to predict the stage of liver fibrosis that could be seen if biopsy was performed. An example of such scoring systems includes Aspartate aminotransferase (AST) to Platelet Ratio (APRI), Fibrosis-4 (FIB4) and NAFLD fibrosis score (NFS) [2].

Hepatic steatosis is usually seen on imaging studies with liver ultrasound, abdominal CT or Proton- Magnetic resonance spectroscopy. If available magnetic resonance elastography and vibration controlled elastography can be used to determine the severity (stage) of fibrosis [3].

In one prospective cohort study of 361 patients with biopsy-proven Nonalcoholic fatty liver disease (NAFLD), daily aspirin use was associated with less risk of progression to fibrosis when compared to nondaily use of Aspirin patients [4]. In the same study, the use of non-aspirin NSAID's was not associated with risk for advanced fibrosis, although this was not the primary endpoint in that study. Another cross-sectional study of 1856 US adult patients with the suspected chronic liver disease showed that aspirin users had lower indices (FIB4, APRI, and NFS) of liver fibrosis [5]. Finally, in a murine model study, the results suggested that 
antiplatelet agents inhibit the platelet-derived growth factor- $\beta$, which activates the hepatic stellate cells responsible for hepatic fibrosis [6].

It is known that aspirin has an irreversible antiplatelet effect, while other NSAID's like ibuprofen have a reversible antiplatelet effect.

Despite the growing incidence and burden of fatty liver disease in the USA $-25 \%$ in general population and $56 \%$ in patients with Diabetes Mellitus type 2 and NASH with Cirrhosis being the second most common cause of liver transplantation in USA, there are no FDA approved medication to prevent the progression of fatty liver disease or reverse the steatosis, fibrosis and inflammation in patients with NAFL-NASH.

The data supporting the potential hepatoprotective effect of NSAID's in patients with fatty liver disease are limited. Therefore, we investigated the association between non-aspirin NSAID's use and risk of fatty liver disease progression to advanced fibrosis.

\section{Methods}

\section{Study Population:}

Patients who we investigate were 18 years of age or older selected from the HCA Healthcare databank system (180 hospitals) with imaging studies that showed fatty liver disease from January 2016 to January 2019.

The imaging studies suggesting fatty liver included Computed tomography (CT), ultra-sonography (US), and magnetic resonance imaging (MRI).

Sunrise Hospital-Mountainview Institutional Review Board approved this study with a waiver of documented informed consent.

All patients were de-identified, and the following variables were collected at their baseline and subsequent visits: demographics (age, gender, and race), Medication use [aspirin, non-aspirin NSAID's (ibuprofen, naproxen, ketoprofen, diclofenac, and indomethacin), Statin use (atorvastatin, simvastatin, and rosuvastatin)], comorbidities [coronary artery disease (CAD), congestive heart failure (CHF), diabetes mellitus type 2(DM 2)], and laboratory parameters required for the calculation of the validated non-invasive indices of liver fibrosis FIB4 and NFS. [7-8].

We did not use magnetic resonance elastography or vibration controlled elastography to determine the magnitude of the stage of liver fibrosis.

We included only patients who had the needed data and variables to calculate the validated indices every year. We also excluded patients with the following history: unclear or heavy alcohol use history, documented history of alcoholic hepatitis, cirrhosis, hemochromatosis, Wilson's disease, polycystic ovary syndrome, obstructive sleep apnea, primary hypothyroidism, viral hepatitis, autoimmune hepatitis, drug-associated hepatitis, and hepatocellular carcinoma.

Based on these criteria, we identified 1347 patients with fatty liver disease.

\section{NSAID's use}

Non-aspirin use (ibuprofen, naproxen, ketoprofen, diclofenac, indomethacin) were confirmed by medical record review at every subsequent hospitalization. Only patients who continued to report use at all hospitalizations from 2016 to 2019 were included.

Radiological diagnosis of fatty liver disease of our patients included one of the following imaging reports by Board Certified Radiologist:

Ultra-sonographic readings of hyperechoic texture, fatty infiltration, increased echogenicity, hepatomegaly, intra-hepatic vascular blurring (sensitivity $85 \%$, specificity $94 \%$ for fatty liver disease with decreased sensitivity in morbid obesity) [9-10-11].

CT readings of reduced attenuation in the liver parenchyma, intrahepatic fat accumulation (Sensitivity $82 \%$, Specificity $100 \%$ for fatty liver disease).

MRI readings of hepatic steatosis (Sensitivity $76 \%$, Specificity $87 \%$ for fatty liver disease) [11-12].

\section{Fibrosis score estimation:}

Advanced fibrosis is a known prognostic factor for poor outcomes, but determining the fibrosis with biopsy is limited because of the invasive nature of the procedure.

Thus, non-invasive screening indices that only require clinical parameters are often used for risk stratification of patients for presence of liver fibrosis.

Using a lower cutoff value of 1.45 , a FIB4 score of less or equal to 1.45 correlates with a negative predictive value of $90 \%$ for liver fibrosis, while a FIB4 cutoff value of greater or equal to 3.25 have $97 \%$ specificity of liver fibrosis. NFS cutoff value of less than or equal to -1.455 has a negative predictive value of $88 \%$ for liver fibrosis, while cutoff value that is greater or equal to 0.676 has a positive predictive value for advanced fibrosis of $82 \%$ [11].

Outcomes:

All included patients had sufficient laboratory testing and clinical data needed to calculate NFS or FIB-4 scores each study year. Patients who had missing clinical or laboratory data were excluded from the cohort. Patients were excluded from analysis if their baseline FIB-4 score was greater or equal to 1.45 or if baseline NFS score was equal or greater than $>0.67$.

The primary outcomes were defined as an increase or decrease from baseline of the fibrotic scores. A secondary analysis was performed in different races, genders, and in patients with diabetes mellitus type 2 .

\section{Statistical analysis:}

Data are expressed as means or numbers $(\mathrm{N})$ with percentages.

To test for normality for age between non-aspirin NSAID's use, we used Shapiro-Wilk Test and the Kruskal-Wallis test. Chi-square statistical approach was used to determine the odds ratio and relative risk of NSAID's use in patients with diabetes, and different gender and ethnicities.

A generalized linear model was used to determine the mean indices score at baseline and at each subsequent year.

\section{Results}

Baseline Characteristics:

Table 1 describes the Baseline characteristics of patients in the study $(n=1347)$. 


\begin{tabular}{cc}
\hline Characteristics & N=1347 (100\%) \\
\hline Mean Age & 40 \\
Gender & \\
Male & $504(37 \%)$ \\
Female & $843(63 \%)$ \\
Mean BMI & 34 \\
Ethnicities & \\
Black & $114(9 \%)$ \\
White & $788(58 \%)$ \\
Other & $445(33 \%)$ \\
NSAIDs use & \\
Aspirin & $138(10 \%)$ \\
Non-aspirin & $1209(90 \%)$ \\
Comorbidities & \\
Non-DM & $1123(83 \%)$ \\
DM & $224(17 \%)$ \\
CHF & $2(0.15 \%)$ \\
CAD & 0 \\
HLD & 0 \\
Mean Baseline Indices & NFS (-1.94), FIB-4 (1.10)
\end{tabular}

Abbreviation: NFS, NAFLD Fibrosis Score; F1B-4, Fibrosis-4; BMI, Body Mass Index; NSAID, nonsteroidal antiinflammatory drug; DM, diabetes mellitus; CHF, congestive heart failure; CAD, coronary artery disease; HLD, hyperlipidemia.

Table1: Baseline clinical and demographic characteristics

Model Analysis of different Parameters:

Table 2 describes the change in validated scores based on different parameters. NSAID's use was associated with lowering of validated indices (pvalue $<0.05$ ).

\begin{tabular}{|c|c|c|}
\hline Parameters & Model Analysis & p-value \\
\hline \multicolumn{2}{|c|}{ NSAIDs Use in Non-DM ( $\mathrm{n}=1123)$ vs. DM Patients (224) } & 0.039 \\
\hline NSAIDs use OR $(95 \% \mathrm{CI})$ & \multicolumn{2}{|l|}{$1.59(1.05-2.44)$} \\
\hline NSAIDs use RR $(95 \% \mathrm{CI})$ & \multicolumn{2}{|l|}{$0.66(0.46-0.95)$} \\
\hline \multicolumn{3}{|c|}{ Change in validated indices } \\
\hline Gender & -0.051 & \multirow{2}{*}{$\begin{array}{c}0.57 \\
<0.0001\end{array}$} \\
\hline NSAIDs use & -0.596 & \\
\hline Black & -0.433 & 0.42 \\
\hline Other & -0.054 & 0.62 \\
\hline Hispanic & -0.277 & 0.0099 \\
\hline \multicolumn{3}{|l|}{ Mean NFS } \\
\hline Gender & -0.068 & 0.44 \\
\hline NSAIDs use & -0.431 & 0.0027 \\
\hline Black & 0.194 & 0.23 \\
\hline Other & -0.234 & 0.03 \\
\hline Hispanic & -0.231 & 0.03 \\
\hline
\end{tabular}

Abbreviation: NFS, NAFLD Fibrosis Score; FIB-4, Fibrosis-4; NSAID, nonsteroidal anti-inflammatory drug; DM, diabetes mellitus

NSAIDs use is associated with lower indices of Liver Fibrosis in patients with nonalcoholic fatty liver disease.

Table 2: Model Analysis of different Parameters. 
Table 3 compares histological staging with corresponding noninvasive scores.

The cohort in this study had a Baseline mean FIB4 of 1.10 and mean NFS of -1.94 which corresponds with no fibrosis or mild fibrosis at baseline.

\begin{tabular}{|l|l|l|}
\hline Histologic Stage & FIB4-Index & NAFLD Score \\
\hline F0-F1 No Fibrosis or Mild Fibrosis & $<1.45$ & $<-1.455$ \\
\hline F1-F2 Moderate Fibrosis & $1.45-3.25$ & $\begin{array}{l}-1.455-0.675 \\
\text { indeterminate score }\end{array}$ \\
\hline F3-F4- Severe Fibrosis or Cirrhosis & $>3.25$ & $>0.675$ \\
\hline
\end{tabular}

Table 3: Fibrosis Severity Scale and corresponding noninvasive staging

Cohort Mean indices at baseline (FIB-4: 1.10; NFS: -1.94).

Noninvasive tests of hepatic fibrosis are used to predict the stage of liver fibrosis that could be seen if biopsy was performed.

Figure 1. Generalized Linear model analysis describes that the patients who used Non- aspirin NSAID's had reduction of the mean fibrotic indices score during each study year ( $\mathrm{p}$ - value of $<0.05$ ).

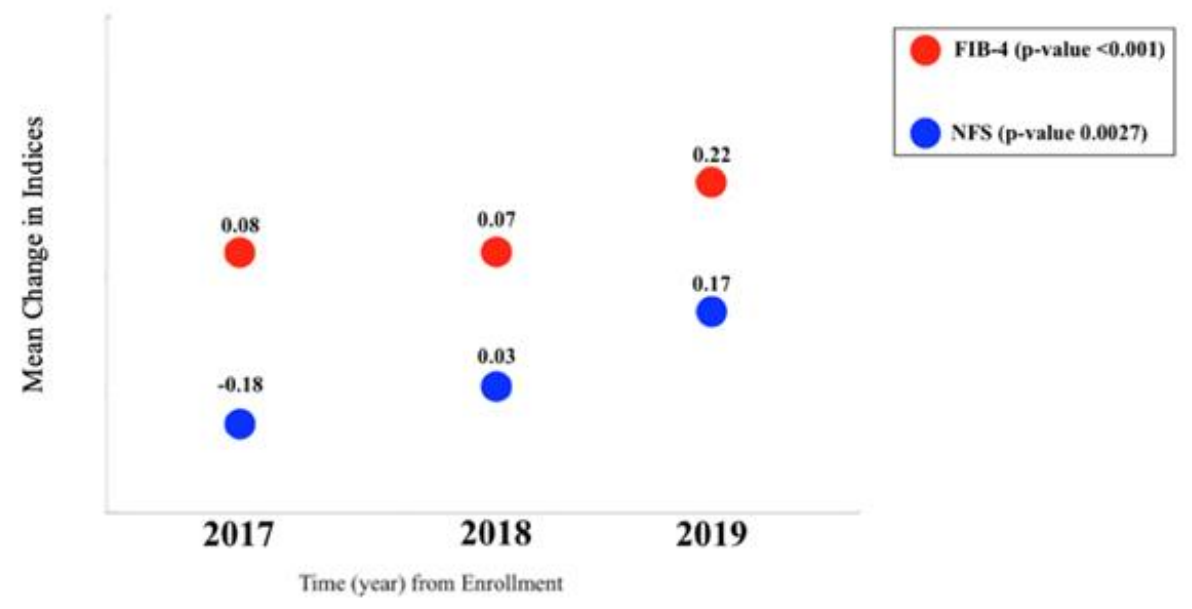

Abbreviations: FIB-4, Fibrosis-4; NFS, NAFLD Fibrosis Score.

Figure 1: Change in validated scores with non-aspirin NSAID's use.

\section{Discussion}

In our retrospective study of patients with fatty liver disease seen on imaging studies described above, non-aspirin NSAID's use was associated with lowering of fibrotic scores-FIB-4 and NFS every year.

Majority of the patients in the study were taking non-aspirin NSAID's for non-cardiac reasons, which suggests that any observed changes were mostly due to non-aspirin effect.

In another prospective study, similar findings were reported in biopsyproven NAFLD patients who were taking aspirin daily [5].

The clinical data needed to fully support the hepato-protective effect of aspirin or other non-aspirin NSAID's is still limited. The current study adds retrospective evidence to support the potential benefit of NSAID's in patients with fatty liver disease.

The observed benefit could be due to mechanisms previously described in murine models, which suggest that platelets stimulate the stellate cells to initiate fibrosis via platelet-derived growth factor (PDGF). NSAID's with reversible or irreversible antiplatelet effect could potentially prevent such hepatic fibrosis by inhibition of PDGF [6].

Given the prevalence of fatty liver disease in the United States and worldwide (10-46\%), NSAID's can potentially benefit those patients.
Thus, further research is needed to better understand and assess the potential antifibrotic benefit versus bleeding and other risks associate with the NSAID's use in patients with fatty liver disease.

We would like to mention that there have being reports in the literature with the usage of Pioglitazone in patients with NAFL/NASH confirmed on liver biopsy in patients with or without Diabetes mellitus type 2. Those reports showed complete resolution of NASH in $51 \%$ of the patients with placebo subtracted difference of $32 \%$ [3]. This improvement correlated with improvement of adipose tissue insulin resistance and increased adiponectin levels.

Similar resolution of NAFL/NASH was observed in patients with Diabetes Mellitus type 2 treated with Liraglutide. 39\% of patients had resolution of NASH. This effects were related to improvement of the body weight and, glycemic control as well as increased insulin sensitivity with Liraglutide [3].

We acknowledge that there might be limitations to this study, which are not limited to the following: 
1. Fatty liver diagnosis was based on imaging reports of different modalities, which could add variability in the observed results.

2. Differences in medication dose and duration of use was not accounted for in this study.

3. The length of the study is not sufficient to fully determine the clinical significance of lowered fibrosis scores.

4. Recall bias and exposure misclassification was not accounted for in this study. However, we tried to minimize this risk by careful examining of the medical records.

5. The presence of other conditions not accounted for or not yet diagnosed may also cause confounding in the observed study results.

6. Non-invasive indices are not the gold standard for evaluating hepatic fibrosis; however, they have demonstrated utility for this purpose.

\section{Conclusion}

In a group of patients with fatty liver disease found on imaging studies, non-aspirin NSAID's use was associated with significant improvement of validated liver fibrotic indices.

Conflict of interests: None to be disclosed for any of the authors.

Funding and Disclaimer: This research was supported (in whole or in part) by HCA Healthcare and/or an HCA Healthcare affiliated entity. The views expressed in this publication represent those of the authors and do not necessarily represent the official views of HCA Healthcare or any of its affiliated entities.

\section{References:}

1. Wong RJ, Liu B, Phuket T. (2017) Significant burden of nonalcoholic fatty liver disease with advanced fibrosis in the US: a cross-sectional analysis of 2011-2014 National Health and Nutrition Examination Survey. Aliment Pharmacol Ther. 46(10):974-980.

2. Hagstrom H, Nasr P, Ekstedt, et al. (2019) Accuracy of noninvasive scoring systems in assessing risk of death and liver-related endpoints in patients with non-alcoholic fatty liver disease. Clinical gastroenterology and hepatology. 17:1148-1156.

3. Barb, Diana. (2020) Pharmacological approaches to the patient with Nonalcoholic Fatty Liver Disease. Endocrine Case management: Meet the professor:121-135.

4. Simon TG, Henson J, Osganian S, Masia R, Chan AT, Chung RT, Corey KE. (2019) Daily Aspirin Use Associated with Reduced Risk for Fibrosis Progression in Patients with Nonalcoholic Fatty Liver Disease. Clin Gastroenterol Hepatol. 17(13):2776-2784.e4.

5. Jiang ZG, Feldbrügge L, Tapper EB, Popov Y, Ghaziani T, Afdhal N, Robson SC, Mukamal KJ. (2016) Aspirin use is associated with lower indices of liver fibrosis among adults in the United States. Aliment Pharmacol Ther 43(6):734-743.

6. Yoshida S, Ikenaga N, Liu SB, Peng ZW, Chung J, Sverdlov DY, Miyamoto M, Kim YO, Ogawa S, Arch RH, Schuppan D, Popov Y. (2014) Extrahepatic platelet-derived growth factor- $\beta$, delivered by platelets, promotes activation of hepatic stellate cells and biliary fibrosis in mice. Gastroenterology. Dec; 147(6):1378-1392.

7. Sterling RK, Lissen E, Clumeck N, Sola R, Correa MC, Montaner J, S Sulkowski M, Torriani FJ, Dieterich DT, Thomas DL, Messinger D, Nelson M. (2006) APRICOT Clinical Investigators. Development of a simple non-invasive index to predict significant fibrosis in patients with HIV/HCV coinfection. Hepatology. 43 (6):1317-1325.

8. Xiao G, Zhu S, Xiao X, Yan L, Yang J, Wu G. (2017) Comparison of laboratory tests, ultrasound, or magnetic resonance elastography to detect fibrosis in patients with non-alcoholic fatty liver disease: A meta-analysis. Hepatology. 66 (5):1486-1501.

9. Hernaez R, Lazo M, Bonekamp S, Kamel I, Brancati FL, Guallar E, Clark JM. (2011) Diagnostic accuracy and reliability of ultrasonography for the detection of fatty liver: a meta-analysis. Hepatology. Sep 2; 54 (3):1082-1090.

10. Cho CS, Curran S, Schwartz LH, Kooby DA, Klimstra DS, Shia J, Munoz A, Fong Y, Jarnagin WR, DeMatteo RP, Blumgart LH, D'Angelica MI. (2007) Preoperative radiographic assessment of hepatic steatosis with histologic correlation. J Am Coll Surg. 206 (3):480-488.

11. Li Q, Dhyani M, Grajo JR, Sirlin C, Samir AE. (2018) Status of imaging in non-alcoholic fatty liver disease. World J Hepatol. 10 (8):530-542.

12. Vernon G, Baranova A, Younossi ZM. (2011) Systematic review: the epidemiology and natural history of non-alcoholic fatty liver disease and non-alcoholic steatohepatitis in adults. Aliment Pharmacol Ther. 34 (3):274-285.
This work is licensed under Creative Commons Attribution 4.0 License
To Submit Your Article Click Here: Submit Manuscript

DOI: $10.31579 / 2640-1045 / 071$
Ready to submit your research? Choose Auctores and benefit from:

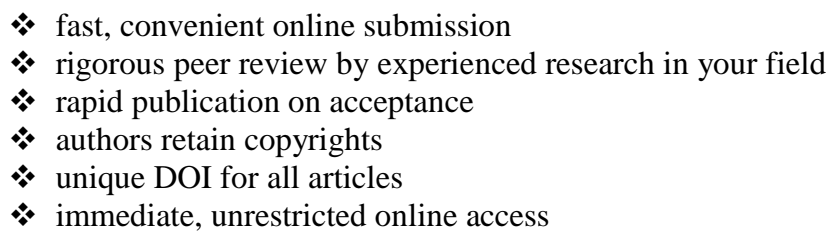

At Auctores, research is always in progress.

Learn more https://auctoresonline.org/journals/endocrinology-anddisorders 